\title{
P21-Driven Multifusion Gene System for Evaluating the Efficacy of Histone Deacetylase Inhibitors by In Vivo Molecular Imaging and for Transcription Targeting Therapy of Cancer Mediated by Histone Deacetylase Inhibitor
}

\author{
Ya-Ju Hsieh*1, Luen Hwu*2, Yi-Chieh Chen ${ }^{2,3}$, Chien-Chih Ke², Fu-Du Chen ${ }^{4}$, Hsin-Ell Wang ${ }^{3}$, Kang-Ping Lin ${ }^{5,6}$, \\ Hsin-Hsien Yeh ${ }^{2,3}$, Chi-Wei Chang ${ }^{2,7}$, and Ren-Shyan Liu ${ }^{2,3,7,8}$ \\ ${ }^{1}$ Department of Medical Imaging and Radiological Sciences, Kaohsiung Medical University, Kaohsiung, Taiwan; ${ }^{2}$ Molecular and \\ Genetic Imaging Core/Taiwan Mouse Clinic, National Comprehensive Mouse Phenotyping and Drug Testing Center, Taipei, Taiwan; \\ ${ }^{3}$ Department of Biomedical Imaging and Radiological Sciences, National Yang-Ming University, Taipei, Taiwan; ${ }^{4}$ Center for Teaching \\ and Learning Resources, Chinese Culture University, Taipei, Taiwan; ${ }^{5}$ Department of Electrical Engineering, Chung Yuan Christian \\ University, Chungli, Taiwan; ${ }^{6}$ Holistic Medical Device Development Center, Chung Yuan Christian University, Chungli, Taiwan; \\ ${ }^{7}$ National PET/Cyclotron Center and Department of Nuclear Medicine, Taipei Veterans General Hospital, Taipei, Taiwan; and \\ ${ }^{8}$ Institute of Clinical Medicine, National Yang-Ming University, Taipei, Taiwan
}

Overexpressed histone deacetylase (HDAC) activity has been linked with tumor initiation and progression that prompt the development of histone deacetylase inhibitors (HDACls) as anticancer agents. $\mathrm{HDACl}$ was reported to be able to activate p21 promoter through the SP1 binding sites in the proximal region of $\mathrm{p} 21_{\mathrm{WAF} 1 / \mathrm{CIP} 1}$ promoter. In this study, we established a p21WAF1/CIP1 promoter-driven triple-fused reporter gene system (p21-3H) to evaluate the efficacy of $\mathrm{HDACl}$ and the ganciclovir (GCV)-mediated anticancer effect contributed by $\mathrm{HDACl}$-induced and p21-driven truncated herpes simplex virus-1 thymidine kinase sr39 mutant (ttksr39) in vitro and in vivo. Methods: The $\mathrm{p} 21-3 \mathrm{H}$ construct was generated and stably or transiently transfected into $\mathrm{H} 1299$ cell lines. These cells were treated with trichostatin A or vorinostat (suberoylanilide hydroxamic acid [SAHA]) to evaluate the activation of $\mathrm{p} 21$ promoter-driven reporter gene expression by in vitro confocal fluorescence microscopy, luciferase assay, 2'-fluoro2 -deoxyarabinofuranosyl-5-ethyluracil ( $\left.{ }^{3} \mathrm{H}-\mathrm{FEAU}\right)$ cellular uptake, in vivo bioluminescence imaging, and $9-\left(4-{ }^{18} \mathrm{~F}-\right.$ fluoro-3-hydroxymethylbutyl) guanine $\left({ }^{18} \mathrm{~F}-\mathrm{FHBG}\right)$ small-animal PET imaging. The therapeutic efficacy on $\mathrm{p} 21-3 \mathrm{H}$-expressing tumor xenografts was assessed by daily administration with SAHA ( $100 \mathrm{mg} / \mathrm{kg}$ intraperitoneally) or GCV (20 mg/kg) for $9 \mathrm{~d}$, followed by tumor volume measurement. Results: On treatment with trichostatin A or SAHA, H1299 cells carrying p21$3 \mathrm{H}$ showed a significant increase of luciferase activity, cellular uptake of ${ }^{3} \mathrm{H}-\mathrm{FEAU}$ (Moravek), and DsRed expression. In vivo tumor xenografts carrying $\mathrm{p} 21-3 \mathrm{H}$ also showed increased luciferase activity by luminescent imaging and enhanced accumulation of ${ }^{18} \mathrm{~F}-\mathrm{FHBG}$ by small-animal PET imaging. Furthermore, when cells transfected with $\mathrm{p} 21-3 \mathrm{H}$ or $\mathrm{p} 21 / \mathrm{Pstl}-3 \mathrm{H}$ (which lacks p53-binding sites) were treated, the increase of luciferase activity was similar in both groups, indicating that $\mathrm{HDACl}$-induced p21 promoter activation is independent of $\mathrm{p} 53$. Both in vitro and in vivo results showed improved therapeutic effect by combined treatment of GCV and

Received May 24, 2013; revision accepted Nov. 13, 2013.

For correspondence or reprints contact: Ren-Shyan Liu, Department of Nuclear Medicine, National Yang-Ming University Medical School, Taipei VGH, 201, Sec. 2, Shih-Pai Rd., Taipei, Taiwan 112.

E-mail: rsliu@vghtpe.gov.tw

${ }^{*}$ Contributed equally to this work.

Published online Mar. 17, 2014.

COPYRIGHT (c) 2014 by the Society of Nuclear Medicine and Molecular Imaging, Inc.
HDACl. Conclusion: We have established an HDACl-inducible, p21-driven reporter system that has the potential for evaluating the anticancer effect of HDACls on cancer cells by multiple molecular imaging modalities. Furthermore, ttksr39 in a p21-3H reporter construct provides a potential combination with thymidine kinase-mediated gene therapy to optimize the therapeutic benefit of HDACl.

Key Words: HSV1-tk; histone deacetylase inhibitor; ${ }^{18} \mathrm{~F}-\mathrm{FHBG}$; 21 ; PET

J Nucl Med 2014; 55:678-685

DOI: 10.2967/jnumed.113.126573

$\mathbf{T}$ umor initiation and progression can evolve from genetic mutations or epigenetic abnormalities (1). The transcription of genes can be regulated by epigenetic modifications including methylation of $\mathrm{CpG}$ islands within promoter regions, acetylation, or deacetylation of histone proteins performed by histone acetyltransferase or histone deacetylase (HDAC), respectively (1-3). These epigenetic modifications delicately organize the chromatin states to maintain cellular homeostasis of proliferation, differentiation, and survival; otherwise, unbalanced epigenetic modifications will lead to inappropriate gene silencing, gene overexpression, or tumorigenesis. HDACs also act on many nonhistone proteins such as transcription factors (e.g., p53, hypoxia-inducible factor 1- $\alpha$, nuclear factor-к B), signal transducers (e.g., signal transducer and activator of transcription 1/3 [STAT1/3]), chaperons (e.g., heat shock protein 90, glucoseregulated protein 78), and cytoskeleton protein (e.g., tubulin) (4). Thus, the status of acetylation has been implicated in physiologic processes including RNA splicing, DNA damage repair, cell cycle control, apoptosis, nuclear transport, actin remodeling, and chaperon function (5).

Eighteen HDAC isoforms have been identified and subdivided into 4 classes (6). Overexpression of HDAC class I (HDAC1, -2, -3, and -8 ) has been clinically associated with prostate cancer, gastric cancer, breast cancer, and colon cancer whereas class II HDAC6 is relevant to elevated messenger RNA and protein level in oral 
squamous cell carcinoma, compared with normal squamous epithelium (7). Even though it is currently unclear whether HDAC overexpression is a consequence of or contributor to tumorigenesis, HDAC overexpression alters the expression and function of tumorassociated proteins that are involved mainly in cell proliferation, migration, angiogenesis, and metastasis $(6,8)$. Besides, patients who had cancer with aberrant HDAC expression showed a positive correlation with poor prognosis $(9,10)$. These clinical observations prompted the development of HDAC inhibitor (HDACI) for anticancer therapies.

HDACIs are capable of interfering with HDAC activity and reversing aberrant epigenetic states associated with cancer (11). They induce apoptosis of cancer cells by regulating proapoptotic and antiapoptotic genes involved in mitochondrial (intrinsic) and death-receptor (extrinsic) $(12,13)$ apoptotic pathways and suppress cell proliferation through activation of cell cycle checkpoints at G1/S or G2/M (12). Moreover, their function in the suppression of angiogenesis and regulation of host immune responses partly contributes to anticancer response. Several structurally diverse HDACIs have been introduced into clinical trials. Some of them specifically target only 1 class of HDACs, whereas some block several classes of HDACs such as suberoylanilide hydroxamic acid (SAHA), which inhibits class I (HDAC1, -2, and -3) and class II (HDAC6).

SAHA, also known as vorinostat, was reported to induce apoptosis in human myelomonocytic cells, myeloma cells, acute T-cell leukemia cells, lymphoma cells (14), and prostate carcinoma $(12,14,15)$. SAHAinduced differentiation was also observed in prostate cancer cells (15). In addition, the antitumor activity of SAHA was well demonstrated in several xenografts including human breast cancer, prostate cancer, and colon cancer (14). Subsequent evaluation of SAHA in clinical trials led to approval by the Food and Drug Administration of the first-in-class HDACI for cutaneous $\mathrm{T}$ cell lymphoma treatment (14). Studies of prostate cancer models showed that SAHA treatment induced transformed cell death or tumor growth inhibition, without prominent toxicity to normal cells or experimental mice $(15,16)$. Recently, Lee et al. showed that SAHAinduced DNA double-strand breaks were accumulated both in normal and in transformed cells, but DNA damage loci were repaired in normal cells only after removal of SAHA, suggesting the selective antitumor activity of SAHA. Furthermore, some DNA repair genes were downregulated in cancer cells after SAHA treatment and resulted in failure of DNA repair (17). The safety, toxicity, and promising response of SAHA have been validated in phase I and II trials of refractory cutaneous T cell lymphoma. However, for other phase II trials of solid tumors-including ovarian, breast, colorectal, non-small cell lung, and head and neck cancers and glioblastoma-SAHA has only moderate effects (18). Combined regimens of SAHA with other chemotherapeutic agents (e.g., taxol, cisplatin) or radiotherapy are currently under investigation in clinical trials $(14,19)$ to achieve better therapeutic response.

Treatment of HDACI was reported to alter the cellular transcription of about $2 \%-10 \%$ of expressed genes $(9,20)$. Glaser et al. identified 8 upregulated genes and 5 downregulated genes in HDACI-treated cells $(20)$. The $\mathrm{p} 21^{\mathrm{WAF} 1 / \mathrm{CIP} 1}$, a cyclin-dependent kinase inhibitor with a $33.9 \pm 7.6$-fold change after HDACI treatment, was the most dominantly upregulated gene. The same phenomenon was observed in hepatoma cells, in which p21 WAF1/CIP1 exhibited greater than a 5-fold increase in gene expression after treatment with trichostatin A (TSA [Sigma-Aldrich]) (21). Consistent with other studies $(22,23)$, these results indicated that $\mathrm{p} 21^{\mathrm{WAF} 1 / \mathrm{CIP} 1}$ is one of the most common genes induced by HDACI. After this cascade regulation, we developed an HDACI-inducible reporter gene-expressing system that consisted of $\mathrm{p} 21^{\mathrm{WAF} 1 / \mathrm{CIP} 1}$ promoter-regulated inframe-fused triple-reporter genes, including monomeric DsRed (DsRedm), firefly luciferase (fluc; note that $f l u c$ refers to the gene and FLUC to the protein), and truncated herpes simplex virus type I thymidine kinase sr39 mutant (ttksr39; note that ttksr39 refers to the gene and tTKSR39 to the protein) (referred to as p21-3H). This design enables us to not only evaluate the efficacy of HDACI-mediated transcriptional targeting but also provide a novel combinational suicide therapy that is mediated by the HDACI-thymidine kinase (TK)ganciclovir (GCV) synergistic effect. The aim of this study was to develop a rapid and sensitive reporter system to monitor the tumor response of HDACI treatment and to enhance the antitumor effect by TK-mediated gene therapy.

\section{MATERIALS AND METHODS}

\section{Plasmid Construction}

p21-3H expression vector, which carries a triple-fused reporter gene controlled by human p21/WAF1 promoter, was constructed. In brief, a human $\mathrm{p} 21$ /WAF1 promoter (positions $-2,479$ to +50 ) was polymerase chain reaction (PCR)-amplified from human genomic DNA with designed primers (hp21 forward: 5'-CCACCAGCTAGCCTTCCTCACATCCTCCTT C-3' and hp21 reverse: 5'-CAGGATCCACAAGGAACTGACTTCGGCA-3'). The PCR product was cloned into $\mathrm{p} 3 \mathrm{H}$ reporter vector, which contains DsRedm, fluc, and ttksr39 to generate p21-3H (24). The p21/PstI-3H was generated by removing p53-binding sites from the $\mathrm{p} 21$ promoter in $\mathrm{p} 21-3 \mathrm{H}$.

\section{Cell Culture}

The non-small cell lung cancer cell line H1299 (CRL5803) and human lung adenocarcinoma epithelial cell line A549 (BCRC60074) were purchased from American Type Culture Collection and Bioresource Collection and Research Center in Taiwan, respectively. These cells were cultured in RPMI-1640 medium (Gibco-Invitrogen) supplemented with L-glutamine, penicillin $(100 \mathrm{U} / \mathrm{mL})$, streptomycin $(100 \mathrm{mg} / \mathrm{mL})$, and $10 \%$ fetal bovine serum at $37^{\circ} \mathrm{C}$ in a humidified atmosphere containing $5 \% \mathrm{CO}_{2}$.

\section{Reagents}

TSA was dissolved in $100 \%$ ethanol at a stock concentration of 1 $\mathrm{mg} / \mathrm{mL}$. SAHA was generously provided by Professor Hsin-Ell Wang and dissolved in dimethyl sulfoxide (DMSO) at a concentration of $50 \mathrm{mM}$. GCV (Sigma-Aldrich) was dissolved in phosphate-buffered saline at a concentration of $40 \mathrm{mg} / \mathrm{mL}$. Culture medium was used for further dilution to proper working concentration for subsequent cell and animal use.

\section{Transient Transfection and Generation of Stable Cell Lines}

For transient transfection, H1299 or A549 cells $\left(1 \times 10^{5}\right)$ were plated in 12-well plates $24 \mathrm{~h}$ before transfection. The transfection mixture for each well was prepared by mixing $1.5 \mu \mathrm{g}$ of DNA and $1.5 \mu \mathrm{L}$ of transfection reagent (Bio-Rad Laboratories) in serum-free medium and then adding the mixture into the cell culture. Six hours after transfection, the medium was replaced with fresh complete medium. A plasmidcontaining Renilla luciferase gene cotransfected with p21-3H construct was used to normalize the transfection efficiency. Subsequent experiments were performed $48-72 \mathrm{~h}$ after transfection. To generate stable cell lines, the transfected cells were trypsinized, and 500 cells were seeded in $10-\mathrm{mm}$ culture dishes. Drug selection was carried out by incubating cells with $700 \mu \mathrm{g} / \mathrm{mL}$ G418 of (Sigma-Aldrich) for approximately $2 \mathrm{wk}$. Survived individual clones were isolated and expanded. 


\section{Laser Confocal Microscopy}

H1299-p21-3H stable cells or wild-type H1299 (H1299-wt) cells were seeded in chamber slides (Lab-Tek) and treated with TSA or SAHA. Twenty-four hours after treatment, cells were washed, permeabilized by $0.5 \%$ Triton-X (Sigma-Aldrich), and stained with 4'6-diamidino-2-phenylindole. Images were acquired using an FV1000 laser confocal microscope (CLSM, Olympus).

\section{Luciferase Reporter Assay}

After cells were seeded and cultured in 12-well plates overnight, cells were treated with TSA $(1 \mu \mathrm{M})$ or ethanol. After 24-h treatment, cells were lysed and assayed for the luciferase activity using a Luciferase Assay System (Promega) and a luminometer (Wallac 1420 Multilabel Counter; PerkinElmer Life Sciences). For transient transfection experiments, luciferase activity in each sample was normalized by Renilla luciferase activity, and the protein concentration of cell lysates was determined by Bradford protein assay reagent (Bio-Rad Laboratories). The normalized FLUC activity is represented by the relative luminescence unit (RLU).

\section{In Vitro Cell Uptake Assay}

Cells were seeded in 12 -well plates $\left(1 \times 10^{5}\right.$ per well $)$ in triplicate and cultured overnight, followed by TSA $(1 \mu \mathrm{M})$ or ethanol treatment for $24 \mathrm{~h}$. Culture medium was then replaced by $0.5 \mathrm{~mL}$ of medium containing 2 '-fluoro-2' -deoxyarabinofuranosyl-5-ethyluracil ( ${ }^{3} \mathrm{H}$-FEAU) $(7.4 \mathrm{kBq}[0.2 \mu \mathrm{Ci}])$. Two hours after incubation, the radioactivity of cells and medium was measured. Medium and $0.5 \mathrm{~mL}$ of phosphatebuffered saline used to rinse the wells were collected in counting vials. Monolayer cells were lysed by $75 \mu \mathrm{L}$ of CytoBuster Protein Extraction Reagent (Novagen) and lysates collected in Eppendorf tubes. After centrifugation at $12,000 \mathrm{rpm}$ for $1 \mathrm{~min}, 100 \mu \mathrm{L}$ of supernatant were transferred to counting vials, and the rest was used to determine total protein concentration. Samples were mixed with $5 \mathrm{~mL}$ of scintillation solution (PerkinElmer), and the radioactivity of ${ }^{3} \mathrm{H}$-FEAU in cells or medium was measured using a $\beta$ counter (Packard Tri-Carb LS Counter; PerkinElmer). Data are presented as the ratio of radioactivity of the cell pellet to that of medium and normalized by total protein concentration ([dpm/g of cells]/[dpm/g of medium]/total protein concentration).

\section{Ethics Statement}

All animal studies were approved by the Institutional Animal Care and Use Committee of National Yang-Ming University and conducted in accordance with the Principles of Laboratory Animal Care and the Guide for the Care and Use of Laboratory Animals (National Science Council, Taiwan, Republic of China).

\section{In Vivo Bioluminescence and Small-Animal PET Imaging}

The $n u / n u$ nude mice (age, 6-8 wk; female) were purchased from National Laboratory Animal Center, Taiwan. H1299-p21-3H stable cells $\left(5 \times 10^{6}\right)$ were subcutaneously injected into the right flank of mice. Two weeks later when tumors reached $8-10 \mathrm{~mm}$ in diameter, mice were subjected to optical bioluminescent imaging at $0,3,6,24$, and $48 \mathrm{~h}$ after intraperitoneal administration of SAHA $(100 \mathrm{mg} / \mathrm{kg})$ or DMSO $(1 \mathrm{~mL} / \mathrm{kg})$. For imaging, animals were anesthetized by $2 \%$ isoflurane gas in oxygen and then intraperitoneally injected with D-luciferin (150 $\mathrm{mg} / \mathrm{kg}$ ). Fifteen minutes later, mice were placed prone in the imaging chamber, and signal was acquired by the optical imaging system (IVIS 50 Imaging System; Xenogen Technology). Bioluminescent signal was recorded as maximum photons $/ \mathrm{s} /$ centimeter $^{2} /$ steradian (photon $/ \mathrm{s} / \mathrm{cm}^{2} / \mathrm{sr}$ ), displayed in pseudocolors and superimposed on the photographic image using Living Image Software (version 2.50; Xenogen Technology) provided by the manufacturer.

For small-animal PET imaging, 9-(4- ${ }^{18}$ F-fluoro-3-hydroxymethylbutyl) guanine $\left({ }^{18} \mathrm{~F}-\mathrm{FHBG}\right)$ was prepared as previously described (25) and used in this study. Tumor-bearing mice were imaged before and after intraperitoneal injection with SAHA $(100 \mathrm{mg} / \mathrm{kg})$ or DMSO (1 mL/ $\mathrm{kg}$ ) using a microPET R4 scanner (Concord Microsystems). In brief, 1 $\mathrm{h}$ after tail vein injection of $7.4 \mathrm{MBq}(200 \mu \mathrm{Ci})$ of ${ }^{18} \mathrm{~F}-\mathrm{FHBG}$, mice were anesthetized by $2 \%$ isoflurane in oxygen and placed prone on the scanning bench. Static images were acquired for $10 \mathrm{~min}$. Post-SAHA treatment imaging was performed $6 \mathrm{~h}$ after SAHA administration. A tumor region of interest was selected and normalized to a contralateralmuscle region of interest to generate a tumor-to-muscle ratio. The imaging process was done by the small-animal PET Manager, (version 2.2.4; Concord Microsystems), provided by the manufacturer.

\section{MTT (3-[4,5-Dimethylthiazol-2-yl]-2,5-Diphenyltetrazolium Bromide) Cell Viability Assay}

Cells were plated in 96-well plates with 5,000 cells per well. One day afterward, cells were treated with $1,2,4$, or $8 \mu \mathrm{M}$ SAHA combined with or without $5 \mu \mathrm{M} \mathrm{GCV}$ (Sigma-Aldrich). After $72 \mathrm{~h}$ of incubation, medium was replaced by $150 \mu \mathrm{L}$ of medium containing $10 \%$ MTT (Sigma-Aldrich) solution, and cells were incubated for $2 \mathrm{~h}$ at $37^{\circ} \mathrm{C}$. To quantify the cell viability, the formazan crystals within cells were solubilized by $100 \mu \mathrm{L}$ of DMSO, and the absorbance (570 and $630 \mathrm{~nm}$ ) was measured by an enzyme-linked immunosorbent assay reader (Power Wave 340; Bio-Tek Instruments, Inc.). Cell survival was determined according to the absorbance of each group and normalized by the absorbance of the nontreated group.

\section{In Vivo Therapy}

Tumors derived from $5 \times 10^{6} \mathrm{H} 1299-\mathrm{p} 21-3 \mathrm{H}$ stable cells were established as described above. Mice were divided into 4 groups: SAHA (100 mg/kg), GCV (20 mg/kg), SAHA plus GCV treated, and control. Drugs were intraperitoneally injected every day for $9 \mathrm{~d}$. Tumor size was measured by digital caliper every day to evaluate the effect of combined therapy with HDACI and GCV in vivo.

\section{Statistical Analysis}

All statistical tests between control and experimental groups were analyzed by paired-samples tests with use of SPSS software (version 10; SPSS Inc.). A $P$ value of less than 0.05 was taken to indicate statistical significance.

\section{RESULTS}

\section{Construction of p21WAF1/CIP1 Promoter-Driven Triple-Fusion Reporter Plasmid}

HDACIs such as TSA are reported to be able to activate p21 promoter through the SP1 binding sites in the proximal region of the p21 promoter $(26,27)$. Thus, a 2.5 -kb fragment of human p21 promoter was cloned into the upstream of a triple-fused reporter gene (DsRedm, fluc, and truncated mutant ttksr39) to generate the construct $\mathrm{p} 21-3 \mathrm{H}$. In the construct $\mathrm{p} 21 / \mathrm{PstI}-3 \mathrm{H}$, a 237-bp fragment containing 2 p53-binding sequences within the p21 promoter was removed to examine the effect of p53 on p21 gene expression (Fig. 1).

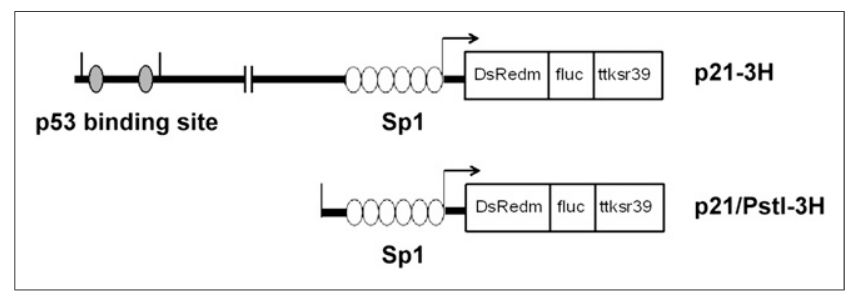

FIGURE 1. Scheme of $\mathrm{p} 21-3 \mathrm{H}$ and $\mathrm{p} 21 / \mathrm{Pstl}-3 \mathrm{H}$ constructs. 


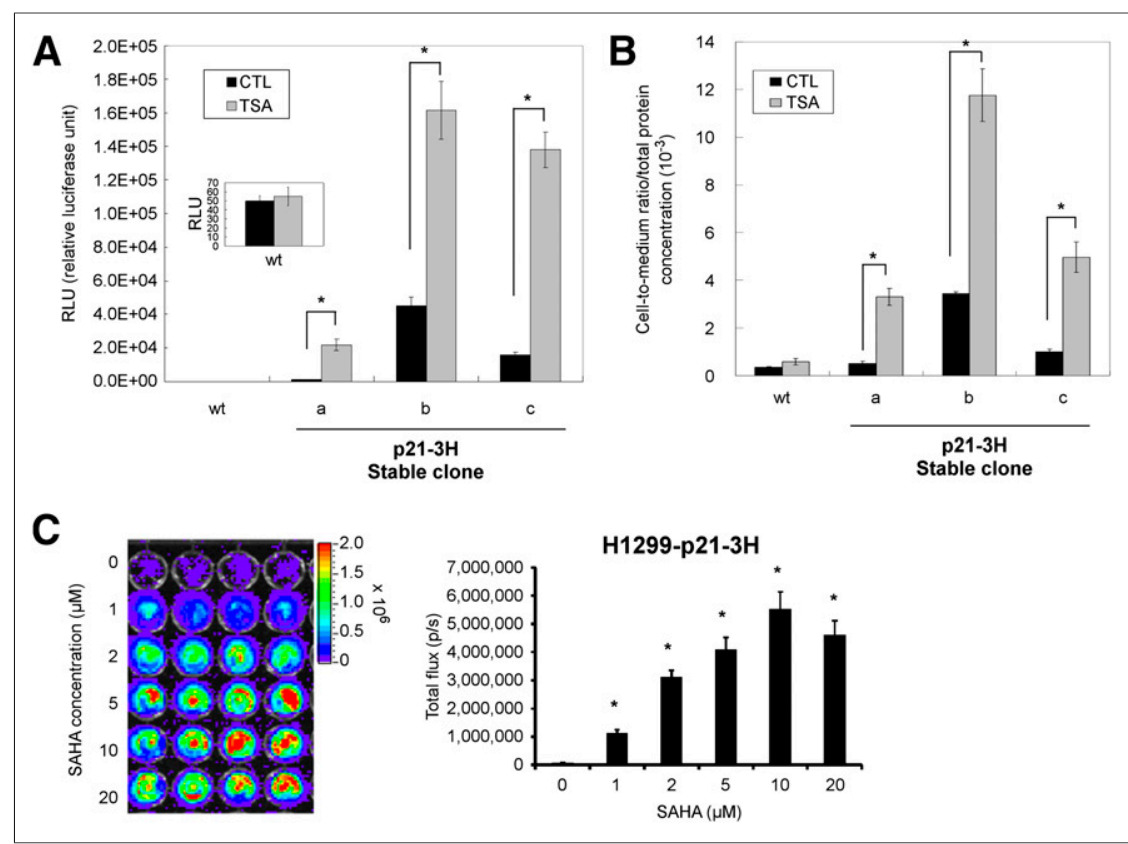

FIGURE 2. Activation of p21 promoter-driven FLUC and ttksr39 activity in H1299-p21-3H stable cells after treatment with TSA or SAHA. fluc assays (A) and ${ }^{3} \mathrm{H}$-FEAU uptake assays (B) were performed to analyze change of FLUC and ttksr39 activity with or without TSA $(1 \mu \mathrm{M})$ treatment for $24 \mathrm{~h}$. Both FLUC and ttksr39 activity significantly increased after TSA treatment. H1299-wt cells were used as control group (CTL). (C) H1299-p21-3H stable cells were treated with 1, 2, 5, 10 , and $20 \mu \mathrm{M}$ SAHA and analyzed by IVIS 50 imaging system. FLUC activity in stable cells increased significantly after SAHA treatment, as compared with cells with no treatment. Total flux represents photons per second. ${ }^{*} P<0.05$.

\section{Establishment and Verification of H1299-p21-3H Stable Cell Line}

To evaluate the influence of HDACI on p21 promoter, we established a H1299 cell line that stably expresses p21-3H gene. H1299 cells were transfected with a $\mathrm{p} 21-3 \mathrm{H}$ construct, followed by treatment with G418 (700 $\mu \mathrm{g} / \mathrm{mL})$ for $2 \mathrm{wk}$. Cell colonies that survived drug

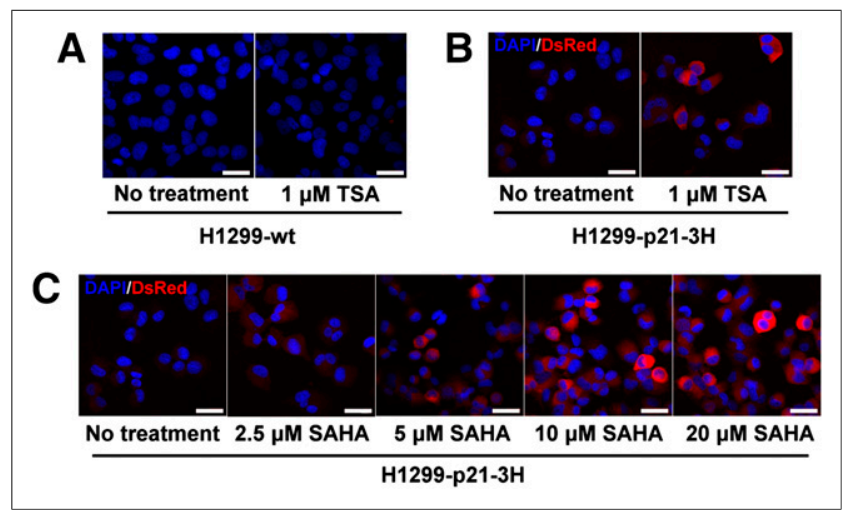

FIGURE 3. Activation of p21 promoter-driven DsRed expression in H1299-p21-3H stable clone cells after treatment with TSA or SAHA. H1299-wt (A) or H1299-p21-3H (B) stable cells were treated without or with $1 \mu \mathrm{M}$ TSA. After treatment, DsRed expression was obviously observed in H1299-p21-3H stable cells, as imaged by confocal laser scanning microscopy. (C) After treatment of a series of concentrations of SAHA $(0,2.5,5,10$, or $20 \mu \mathrm{M})$, expression of DsRed in H1299-p21-3H stable cells increased after increase of SAHA concentration. DAPI (4'6diamidino-2-phenylindole) was used for nuclear staining (blue). Bars in each micrograph represent $40 \mu \mathrm{m}$. selection were subjected to analysis for their p21-3H expression. Expression of p21-3H was observed in 3 (clones a, b, and c) of 25 clones after treatment with HDACI, and clone c was selected for subsequent experiments.

fluc assay showed that clone a had the lowest basal level of FLUC activity and showed a $19.6 \pm 3.0$-fold increase after treatment of TSA. In contrast, clone b inherently had the highest basal gene expression of FLUC among these clones and showed a slight increase by TSA treatment $(3.6 \pm$ 0.4-fold). Clone $\mathrm{c}$ showed intermediate FLUC activity in the absence of TSA and about an $8.8 \pm 0.7$-fold increase by TSA triggering. The gene expression activity of H1299-wt cells was not altered by TSA treatment (Fig. 2A).

TSA induced ${ }^{3} \mathrm{H}$-FEAU uptake in all 3 stably transduced cell clones, which showed a significant increase of ${ }^{3} \mathrm{H}$-FEAU uptake, compared with that in H1299-wt cells. After TSA treatment, $3.4 \pm 0.3-$, $5.0 \pm 0.6-$, and $6.5 \pm 0.7$-fold increases of ${ }^{3} \mathrm{H}-\mathrm{FEAU}$ uptake were observed in clones b, c, and a, respectively, compared with uptake in the same cells before TSA treatment (Fig. 2B). In addition to TSA, we also demonstrated the effect of SAHA in H1299-p21-3H cells using an IVIS 50 imaging system (Xenogen). As shown in Figure 2C, FLUC activity increased after treatment with different concentrations of SAHA, and the $10 \mu \mathrm{M}$ of SAHA showed the highest induction effect of $72.0 \pm 12.5$-fold $(P<0.005)$.

\section{Transcriptional Activity of p21 Promoter Induced by HDACI}

The H1299-p21-3H cells and H1299-wt cells were treated with $1 \mu \mathrm{M}$ TSA for $24 \mathrm{~h}$. Fluorescent microscopic analysis of H1299p21-3H cells revealed increased DsRed fluorescent signal in the cytoplasm in the presence of TSA (Fig. 3A), whereas the H1299wt did not show any red fluorescence in the condition with or without TSA (Fig. 3B). A similar result was observed in H1299p21-3H cells treated with SAHA, indicating that the p21 promoter was TSA- or SAHA-inducible (Fig. 3C). Moreover, SAHA induces the transcriptional activity of $\mathrm{p} 21$ promoter in a dose-dependent manner.

\section{HDACls Induced p21-Driven Gene Expression In Vivo}

TSA has been demonstrated to induce $\mathrm{p} 21^{\mathrm{WAF} 1 / \mathrm{CIP} 1}$ promoter activity by $\mathrm{p} 21-3 \mathrm{H}$. However, TSA is not suitable for clinical use because of its neurotoxicity. Considering the clinical application and side effects on normal cells, the Food and Drug Administration-approved HDACI SAHA, which is a TSA analog, was used in the current animal studies.

After D-luciferin injection (3, 6, 24, and $48 \mathrm{~h}$ ), in vivo bioluminescence imaging was performed on mice bearing H1299-p21-3H and H1299-wt xenografts (Fig. 4A). The result of FLUC activity showed that SAHA treatment significantly induced the transcription activity of p21 $1^{\mathrm{WAF} 1 / \mathrm{CIP} 1}$ in H1299-p21-3H tumors at all time points, compared with that in tumors treated with DMSO. FLUC activity within the tumor reached the maximum value $6 \mathrm{~h}$ after SAHA treatment (Fig. 4B). 


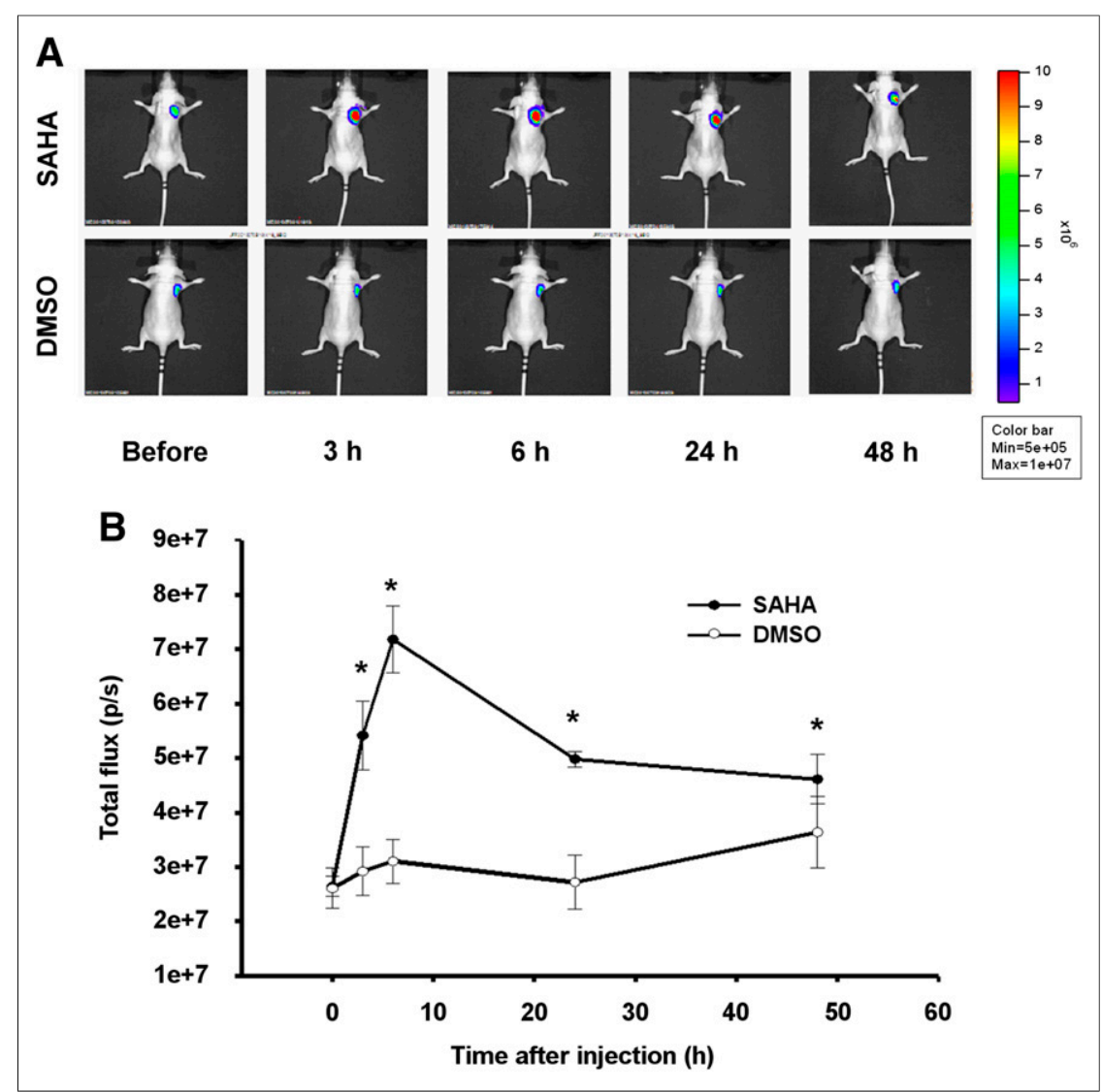

FIGURE 4. In vivo luminescence imaging of activation of p21-driven fluc gene expression. (A) H1299-p21-3H stable cells were implanted and grown subcutaneously on nude mice. Before and $3,6,24$, and $48 \mathrm{~h}$ after intraperitoneal administration of SAHA $(100 \mathrm{mg} / \mathrm{kg})$ or DMSO $(1 \mathrm{~mL} / \mathrm{kg})$, mice were subjected to in vivo luminescence imaging after D-luciferin intraperitoneal injection $(150 \mathrm{mg} / \mathrm{kg}$ ). (B) Total flux (photons/s) in region of interest over tumor region was analyzed using Living ImageR 2.50 software. Each point represents mean \pm SE $(n=4) .{ }^{*} P<0.05$.

Mice were also subjected to small-animal PET imaging to determine the tTKSR39 catalytic activity by measuring the accumulation of ${ }^{18} \mathrm{~F}-\mathrm{FHBG}$ within H1299-p21-3H tumors. A significant increase of ${ }^{18}$ F-FHBG accumulation in H1299-p21-3H tumors was observed $6 \mathrm{~h}$ after SAHA treatment, compared with that in tumors treated with DMSO or compared with that in the same tumors before SAHA

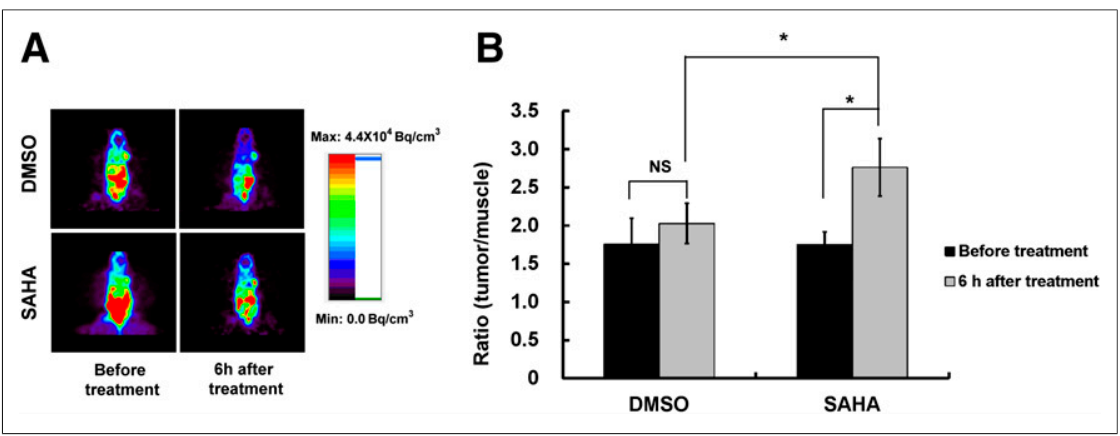

FIGURE 5. In vivo imaging of activation of p21-driven ttksr39 gene expression. (A) Mice bearing $\mathrm{H} 1299-\mathrm{p} 21-3 \mathrm{H}$ tumors were subjected to small-animal PET imaging using ${ }^{18} \mathrm{~F}-\mathrm{FHBG}$ before and $6 \mathrm{~h}$ after SAHA (100 mg/kg) or solvent DMSO (1 mL/kg) injection. (B) Specific ${ }^{18} \mathrm{~F}-\mathrm{FHBG}$ accumulation, which represents expression and activity of TK in tumor region, was analyzed and normalized by activity in contralateral muscle region (tumor-to-muscle ratio). Each bar represents mean $\pm \mathrm{SD}(n=4)$. ${ }^{\star} P<0.05$. Max $=$ maximum; Min $=$ minimum; $\mathrm{NS}=$ not significant. treatment (Fig. 5). The tumor-to-muscle ratio of ${ }^{18} \mathrm{~F}-\mathrm{FHBG}$ accumulation in mice treated with SAHA was about 1.58 times higher than that in mice before SAHA treatment. However, only a 1.15 times change was observed in the control group (Fig. 5B). Slight induction of $\mathrm{p} 21^{\mathrm{WAF} 1 / \mathrm{CIP} 1}$ expression by DMSO treatment in a control group was reported in an earlier study (28).

\section{p53-Independent Induction of p21 WAF1/CIP1 Expression}

To verify that SAHA activates the p21 WAF1/CIP1 promoter through Sp1 sites rather than a 553 -dependent pathway, we generated a construct $\mathrm{p} 21 / \mathrm{PstI}-3 \mathrm{H}$ by removing the p53-binding sequence from $\mathrm{p} 21 / 3 \mathrm{H}$ (Fig. 1). Luciferase assay was performed on H1299 (p53 null) and A549 (p53 wild-type) cells after transient transfection of $\mathrm{p} 21-3 \mathrm{H}$ or p21/PstI-3H and SAHA treatment. Introduction of both constructs induced an approximately 4 and 8 times increase in FLUC activity in A549 and H1299 cells, respectively, after treatment with SAHA for $24 \mathrm{~h}$ (Fig. 6). Although p21/PstI-3H lacks the p53-binding site, the increase of FLUC activity after SAHA treatment was almost the same in p21/PstI-3H and p21-3Htransfected cells. These results suggested that SAHA activated the $\mathrm{p} 21^{\mathrm{WAF} 1 / \mathrm{CIP} 1}$ promoter through a p53-independent pathway regardless of the p53 status of cell lines.

\section{Evaluation of Enhanced Cytotoxicity by GCV Treatment In Vitro and In Vivo}

In our design, tTKSR39 expression is tightly regulated by p21 promoter and can be effectively upregulated by SAHA treatment (Fig. 5). HSV1-TK has been widely used as an imaging reporter and a suicide therapeutic gene when administered with the prodrug GCV. Thus, it is expected that TK-GCV can enhance the effect of SAHA treatment on cancer. To know whether combined treatment with SAHA and GCV enhance the cytotoxicity, H1299-p21-3H or H1299-wt cells were treated with SAHA alone or together with GCV $(5 \mu \mathrm{M})$, and then the cell viability was determined by MTT assay. In the groups treated with SAHA alone, both H1299-wt and H1299-p21-3H cells showed similar cell viability (Fig. 7A). Combined treatment of SAHA and GCV did not induce any improvement of cytotoxicity in H1299-wt cells but significantly enhanced cell death in H1299-p21-3H cells. Notably, when treated with $5 \mu \mathrm{M} \mathrm{GCV}$ and without SAHA, H1299-p21-3H cells showed a cell viability of $0.57 \pm 0.01$ because a certain level of ttksr39 expression was induced by the basal transcriptional activity of p21 promoter. These data showed that therapeutic effect contributed by combined treatment 


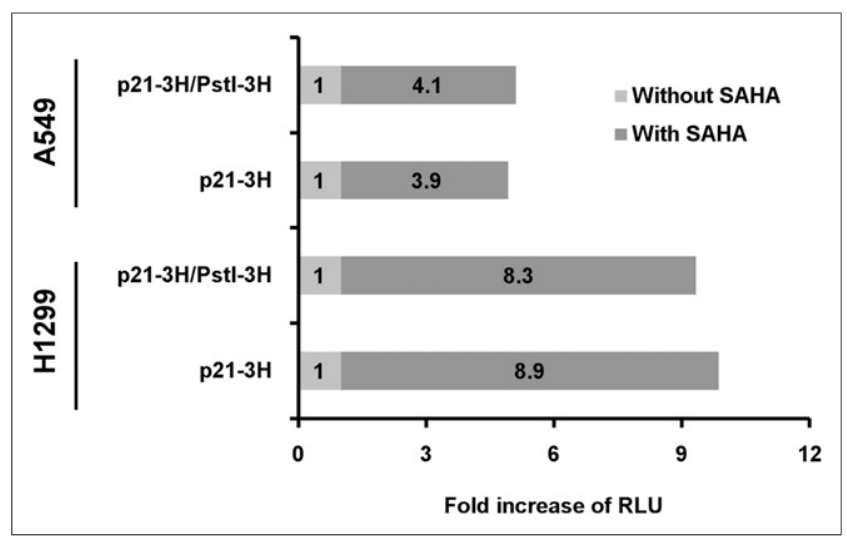

FIGURE 6. HDACl-mediated activation of p21promoter activity is p53-independent. H1299 (p53 null) and A549 (p53 wild-type) cells were transiently transfected with $\mathrm{p} 21-3 \mathrm{H}$ or $\mathrm{p} 21 / \mathrm{Pstl}-3 \mathrm{H}$ construct. After treatment of $2 \mu \mathrm{M}$ SAHA for $24 \mathrm{~h}$, cells were lysed for further luciferase assay. Luciferase activity increased significantly in both transfected A549 and H1299 cells treated with SAHA (dark gray), compared with those without SAHA treatment (light gray). Increase of luciferase activity was observed in A549 cells transfected with p21-3H or p21/Pstl-3H (4.1- and 3.9-fold, respectively) after SAHA treatment. Increase was observed in $\mathrm{H} 1299$ cells transfected with $\mathrm{p} 21-3 \mathrm{H}$ or $\mathrm{p} 21 / \mathrm{Pstl}-3 \mathrm{H}$ (8.3and 8.9 -fold, respectively). RLU $=$ relative luminescence unit.

with SAHA and GCV was more effective than an individual regimen.

We also treated H1299-p21-3H tumor-bearing mice with SAHA and GCV to evaluate the antitumor effect. According to the results shown in Figure 4B, the maximal fold increase of FLUC activity in H1299-p21-3H tumors was observed $6 \mathrm{~h}$ after SAHA injection. Thus, in the following animal study, GCV was given to mice $6 \mathrm{~h}$ after SAHA administration. On treatment with SAHA alone or GCV alone, H1299-p21-3H tumor volume increased approximately 3-fold in $9 \mathrm{~d}$, whereas tumors treated with DMSO showed a 9-fold increase in volume (Fig. 7B). After combined treatment with SAHA and GCV, tumor growth was significantly inhibited. These results clearly demonstrated that HDACI-induced ttksr39 expression is a promising combination candidate in HDACI anticancer treatment.

\section{DISCUSSION}

Overexpressed HDAC activity has been linked with the condensed chromatin structure and results in repressed gene transcription through an epigenetic process. Because the epigenetic alterations are involved in cancer initiation and progression, HDAC seems to be an ideal target to be inhibited (6). HDAC inhibitors are a promising new therapeutic drug that causes cell cycle arrest and promotes cell differentiation and apoptosis in several cancer cell types, and several agents are in clinical trials. However, current clinical trials reveal that the therapeutic effect of HDACIs as a single drug is limited in most solid tumors. TSA was discovered early and has been widely used in studying the function and mechanism of HDACIs because of its powerful inhibition of tumor growth. In this study, we used TSA to test the p21-3H platform initially. However, neurocytotoxic effects occurred when a high dosage of TSA was used. Moreover, the metabolic inactivation of TSA makes it inactive in vivo and unable to inhibit human melanoma tumor growth (29). When clinical usefulness was taken into consideration, SAHA, an analog of TSA, was chosen for our experiments.

$\mathrm{p} 21 \mathrm{Waf} 1 / \mathrm{Cip} 1 / \mathrm{Sdi1}$ is thought to play an important role in tumor suppression because of its ability to inhibit cell cycle progression and DNA replication after DNA damage (30). The transcriptional activation of the $\mathrm{p} 21$ in response to a variety of stressful stimuli can be upregulated by p53 (e.g., DNA damage or mutant RAS) or mediated by a p53-independent mechanism (e.g., TGFß or mimosine). HDACI treatment causes hyperacetylation of histone $\mathrm{H} 3$ and $\mathrm{H} 4$ at the $\mathrm{p} 21^{\mathrm{WAF} 1 / \mathrm{CIP} 1}$ promoter, which can recruit transcriptional factors (RNA polymerase II, p300/histone acetyltransferase, Sp1 and Sp3) to activate $\mathrm{p} 21^{\mathrm{WAF} 1 / \mathrm{CIP} 1}$ expression (22). In animal experiments, spontaneous or chemical carcinogen-induced tumors were more easily formed in p21-deficient mice in comparison with wild-type mice, suggesting that p21 may be a tumor suppressor. It seems that the anticancer activity of HDACI and HDACI-induced tTKSR39 expression through p21 activation might lead to tumor suppression in a synergistic manner (30). However, several oncogenic functions of p21 (e.g., genome destabilization, antiapoptosis, and overexpression of secreted antiapoptotic and mitogenic factors) have been reported in tumor cells under certain conditions depending on cellular context, localization of p21, and circumstances (31). p21 can protect tumor cells from apoptosis because an active cell cycle, which is sensitive to anticancer drugs, is inhibited by p21 to arrest the cell cycle and to induce a senescencelike state in cancer cells (32). Therefore, the exact function of $\mathrm{p} 21$ induced by HDACI in cancer therapy needs further investigation. Despite the uncertain role of p21 in cancer, many studies pointed out the ability of SAHA to induce p21 expression, which provides a 
rational design for our p21 promoter-controlled triple-fusion reporter construct.

In the current study, H1299-p21-3H showed remarkable activity of the reporter gene in response to SAHA or TSA, suggesting p21$3 \mathrm{H}$ could be used as an HDACI indicator. The induction effect of SAHA in transcriptional activity of $\mathrm{p} 21$ promoter was in a dosedependent manner and changed with time in xenografts. Thus, p21-3H can be used as a convenient and real-time platform for monitoring and evaluating the effects of different HDACIs by a molecular imaging modality. Moreover, the optimal working concentration of HDACI and duration of acetylation of target protein, which contribute to the therapeutic efficacy of HDACI, can also be studied on this platform. The activation of p21 promoter by SAHA appears to be not only an efficient but also a rapid process. When $\mathrm{p} 21-3 \mathrm{H}$-bearing mice were treated with SAHA, significant FLUC activity was induced within $3 \mathrm{~h}$ and the induction effect of SAHA persisted for at least $24 \mathrm{~h}$. Because the drug administration in clinical studies using SAHA is always once a day $(33,34)$, the therapeutic effect based on ttksr39 should be efficiently induced and maintained until the termination of the SAHA regimen. The FLUC activity, which was decreasing gradually $6 \mathrm{~h}$ after SAHA administration, might be due to the short half-life of FLUC and SAHA (18). Another possibility might be that SAHA showed a therapeutic effect in reducing the number of cancer cells and consequently decreased reporter gene signals.

The transcription of p21 has been extensively studied and was shown to be mediated by p53-dependent and p53-independent mechanisms. Considering that the mutations in p53 occur in at least $50 \%$ of human tumors and loss of p53 function in malignant tumors is highly associated with drug resistance, the influence of p53 on the p21-3H construct needed to be verified. Our result showed that the induction activity of SAHA does not depend on the p53 status of cancer cells. It is consistent with most of the previous studies that HDACI-induced p21 expression is p53-independent (35). Through this mechanism, HDACIs can exert antitumor activity both in p53wild-type and in p53-deficient cancer cells.

Several studies of $\mathrm{p} 21^{\mathrm{WAF} 1 / \mathrm{CIP} 1}$ regulation were only in vitro (31). A transgenic mouse model carrying a p21-fluc gene construct has been established to investigate the endogenous p21 expression in vivo using a quick and cost-effective optical imaging modality (36). However, the FLUC activity cannot be evaluated by cellular level using microscopy. In addition, most of the optical imaging does not provide optimal tomographic information and quantitative data as compared with 3-dimensional radionuclide imaging techniques (i.e., PET, SPECT) and is limited to application in humans. To overcome these shortcomings and to combine the advantage of fluorescence, bioluminescence, and radionuclide imaging techniques, we fused DsRedm with fluc and ttksr39 to generate a triple-fused reporter gene, and all functions of each reporter gene have been verified in vitro and in vivo $(24,37)$. This multifunctional design has provided a powerful HDACI-related anticancer drug screening platform, which was later demonstrated by Roche R\&D Center (China) Ltd. (38). In this report, p21 and klf2 promoter coupled with a luciferase reporter gene system provided a cell-based readout for the inhibition of HDACs associated either with the p21 or with the klf2 promoter. In this cited study (38), drug selectivity is based on the readout of p21/klf2. klf 2 expression represents an undesirable pharmacologic effect for an HDACI treatment. In our design, we aimed to extend the screening capability to in vivo application. $f l u c$ and $t t k s r 39$ are readily the reporters monitored by in vivo imaging modalities and therefore offer the preclinical value in in vivo anticancer drug testing. Moreover, ttksr39 is not only a reporter gene in terms of molecular imaging but also a therapeutic gene that makes the tumor cell susceptible to being killed by GCV. Because the level of TK expression affects the therapeutic efficacy of the TK-GCV system and the doses of GCV, which contribute to immunosuppressive side effects (39), some researchers have tried to induce the promoter activity by radiation and hypoxia (40). In the current study, we used HDACIs as transcriptional inducers to regulate target gene expression (p21), and a better therapeutic effect was observed using cotreatment with SAHA and GCV prodrug than SAHA treatment alone in vitro and in vivo. Because of the absence of pleiotropic effects and an artificial or complicated experimental setting, using HDACI to manipulate gene expression would be superior to steroid hormones or a tet system (41).

\section{CONCLUSION}

We have demonstrated the effective induction of p21-controlled DsRedm protein, FLUC, and ttksr39 using SAHA. This triple-fused reporter gene construct provides a high-throughput platform for estimating the efficacy and screening of drugs that can induce p21 expression of HDACIs using multiple molecular imaging modalities. Moreover, the $\mathrm{p} 21-3 \mathrm{H}$ construct may provide a potentially feasible HDACI-inducible TK/GCV gene therapy that can improve the antitumor effect of SAHA.

\section{DISCLOSURE}

This research was supported by the grants NSC102-2314-B-010038-MY3, NSC102-2627-M-010-003, and NSC 102-2221-E-033003-MY3 (National Science Council, Taiwan); MOHW103-TD-B111-02 (Department of Health); and V101A-032, V102A-035, and V103C-132 (Taipei Veterans General Hospital). No other potential conflict of interest relevant to this article was reported.

\section{ACKNOWLEDGMENTS}

We thank the Molecular and Genetic Imaging Core, Taiwan Mouse Clinic, which is funded by the National Research Program for Biopharmaceuticals (NSC 102-2325-B-001-042, NRPB) at the National Science Council (NSC) of Taiwan, for technical support and Ms. Tsuey-Ling Jan for the assistance of preparing the manuscript.

\section{REFERENCES}

1. Jones PA, Baylin SB. The epigenomics of cancer. Cell. 2007;128:683-692.

2. Thiagalingam S, Cheng KH, Lee HJ, Mineva N, Thiagalingam A, Ponte JF. Histone deacetylases: unique players in shaping the epigenetic histone code. Ann NY Acad Sci. 2003;983:84-100.

3. Esteller M. DNA methylation and cancer therapy: new developments and expectations. Curr Opin Oncol. 2005;17:55-60.

4. Buchwald M, Kramer OH, Heinzel T. HDACi: targets beyond chromatin. Cancer Lett. 2009;280:160-167.

5. Choudhary C, Kumar C, Gnad F, et al. Lysine acetylation targets protein complexes and co-regulates major cellular functions. Science. 2009;325:834-840.

6. Glozak MA, Seto E. Histone deacetylases and cancer. Oncogene. 2007;26:54205432 .

7. Weichert W. HDAC expression and clinical prognosis in human malignancies. Cancer Lett. 2009;280:168-176.

8. Xu WS, Parmigiani RB, Marks PA. Histone deacetylase inhibitors: molecular mechanisms of action. Oncogene. 2007;26:5541-5552.

9. Mitsiades CS, Mitsiades NS, McMullan CJ, et al. Transcriptional signature of histone deacetylase inhibition in multiple myeloma: biological and clinical implications. Proc Natl Acad Sci USA. 2004;101:540-545. 
10. Zhang Z, Yamashita H, Toyama T, et al. HDAC6 expression is correlated with better survival in breast cancer. Clin Cancer Res. 2004;10:6962-6968.

11. Baylin SB, Ohm JE. Epigenetic gene silencing in cancer: a mechanism for early oncogenic pathway addiction? Nat Rev Cancer. 2006;6:107-116.

12. Bolden JE, Peart MJ, Johnstone RW. Anticancer activities of histone deacetylase inhibitors. Nat Rev Drug Discov. 2006;5:769-784.

13. Frew AJ, Johnstone RW, Bolden JE. Enhancing the apoptotic and therapeutic effects of HDAC inhibitors. Cancer Lett. 2009;280:125-133.

14. Richon VM, Garcia-Vargas J, Hardwick JS. Development of vorinostat: current applications and future perspectives for cancer therapy. Cancer Lett. 2009;280: 201-210.

15. Butler LM, Agus DB, Scher HI, et al. Suberoylanilide hydroxamic acid, an inhibitor of histone deacetylase, suppresses the growth of prostate cancer cells in vitro and in vivo. Cancer Res. 2000;60:5165-5170.

16. Piekarz RL, Bates SE. Epigenetic modifiers: basic understanding and clinical development. Clin Cancer Res. 2009;15:3918-3926.

17. Lee JH, Choy ML, Ngo L, Foster SS, Marks PA. Histone deacetylase inhibitor induces DNA damage, which normal but not transformed cells can repair. Proc Natl Acad Sci USA. 2010;107:14639-14644.

18. Miller CP, Singh MM, Rivera-Del Valle N, Manton CA, Chandra J. Therapeutic strategies to enhance the anticancer efficacy of histone deacetylase inhibitors. J Biomed Biotechnol. 2011;2011:514261.

19. Shabason JE, Tofilon PJ, Camphausen K. HDAC inhibitors in cancer care. Oncology. 2010;24:180-185.

20. Glaser KB, Staver MJ, Waring JF, Stender J, Ulrich RG, Davidsen SK. Gene expression profiling of multiple histone deacetylase (HDAC) inhibitors: defining a common gene set produced by HDAC inhibition in T24 and MDA carcinoma cell lines. Mol Cancer Ther. 2003;2:151-163.

21. Chiba T, Yokosuka O, Arai M, et al. Identification of genes up-regulated by histone deacetylase inhibition with cDNA microarray and exploration of epigenetic alterations on hepatoma cells. J Hepatol. 2004;41:436-445.

22. Richon VM, Sandhoff TW, Rifkind RA, Marks PA. Histone deacetylase inhibitor selectively induces p21WAF1 expression and gene-associated histone acetylation. Proc Natl Acad Sci USA. 2000;97:10014-10019.

23. Sasakawa Y, Naoe Y, Inoue T, et al. Effects of FK228, a novel histone deacetylase inhibitor, on human lymphoma U-937 cells in vitro and in vivo. Biochem Pharmacol. 2002;64:1079-1090.

24. Hwu L, Lin C-F, Hwang A-L, et al. Stably quad-fused reporter gene expressed homogenous cancer cell xenograft for in vivo optical and microPET imaging [abstract]. J Nucl Med. 2008;49(suppl 1):167P.

25. Chang CW, Lin M, Wu SY, et al. A high yield robotic synthesis of 9-(4-[ $\left.{ }^{18} \mathrm{~F}\right]-$ fluoro-3-hydroxymethylbutyl)guanine $\left(\left[{ }^{18} \mathrm{~F}\right] \mathrm{FHBG}\right)$ and $9-\left[3-\left[{ }^{18} \mathrm{~F}\right]\right.$ fluoro-1-hydroxy2-propoxy)methyl $]$ guanine $\left(\left[{ }^{18} \mathrm{~F}\right]\right.$ FHPG) for gene expression imaging. Appl Radiat Isot. 2007;65:57-63.
26. Nakano K, Mizuno T, Sowa Y, et al. Butyrate activates the WAF1/Cip1 gene promoter through Sp1 sites in a p53-negative human colon cancer cell line. J Biol Chem. 1997;272:22199-22206.

27. Sowa Y, Orita T, Minamikawa S, et al. Histone deacetylase inhibitor activates the WAF1/Cip1 gene promoter through the Sp1 sites. Biochem Biophys Res Commun. 1997;241:142-150

28. Ponzio G, Loubat A, Rochet N, et al. Early G1 growth arrest of hybridoma B cells by DMSO involves cyclin D2 inhibition and p21[CIP1] induction. Oncogene. 1998; 17:1159-1166.

29. Qiu L, Kelso MJ, Hansen C, West ML, Fairlie DP, Parsons PG. Anti-tumour activity in vitro and in vivo of selective differentiating agents containing hydroxamate. Br J Cancer. 1999;80:1252-1258.

30. Ocker M, Schneider-Stock R. Histone deacetylase inhibitors: signalling towards p21cip1/waf1. Int J Biochem Cell Biol. 2007;39:1367-1374.

31. Abbas T, Dutta A. p21 in cancer: intricate networks and multiple activities. Nat Rev Cancer. 2009;9:400-414.

32. Roninson IB. Oncogenic functions of tumour suppressor $\mathrm{p} 21^{\text {Waf1/Cip1/Sdi1: asso- }}$ ciation with cell senescence and tumour-promoting activities of stromal fibroblasts. Cancer Lett. 2002;179:1-14.

33. Lane AA, Chabner BA. Histone deacetylase inhibitors in cancer therapy. J Clin Oncol. 2009;27:5459-5468.

34. Rasheed WK, Johnstone RW, Prince HM. Histone deacetylase inhibitors in cancer therapy. Expert Opin Investig Drugs. 2007;16:659-678.

35. Huang L, Sowa Y, Sakai T, Pardee AB. Activation of the $\mathrm{p} 21$ WAF1/CIP1 promoter independent of $\mathrm{p} 53$ by the histone deacetylase inhibitor suberoylanilide hydroxamic acid (SAHA) through the Sp1 sites. Oncogene. 2000;19:5712-5719.

36. Ohtani N, Imamura Y, Yamakoshi K, et al. Visualizing the dynamics of p21 Waf1/ Cip1 cyclin-dependent kinase inhibitor expression in living animals. Proc Natl Acad Sci USA. 2007;104:15034-15039.

37. Chen Y-C, Hwu L, Lin K-H, Wang H-E, Liu R-S. Histone deacetylase inhibitor inducible gene expression for drugs screening and suicide gene therapy [abstract]. J Nucl Med. 2010; 51(suppl 2):200P.

38. Wong JC, Guo L, Peng Z, et al. Application of p21 and klf2 reporter gene assays to identify selective histone deacetylase inhibitors for cancer therapy. Bioorg Med Chem Lett. 2011;21:110-116.

39. Black ME, Kokoris MS, Sabo P. Herpes simplex virus-1 thymidine kinase mutants created by semi-random sequence mutagenesis improve prodrug-mediated tumor cell killing. Cancer Res. 2001;61:3022-3026.

40. Wang WD, Chen ZT, Li R, Li DZ, Duan YZ, Cao ZH. Enhanced efficacy of radiation-induced gene therapy in mice bearing lung adenocarcinoma xenografts using hypoxia responsive elements. Cancer Sci. 2005;96:918-924.

41. Gingrich JR, Roder J. Inducible gene expression in the nervous system of transgenic mice. Annu Rev Neurosci. 1998;21:377-405. 